\title{
Rheological analysis on non-Newtonian wire coating
}

\author{
Azad Hussain $^{1} \cdot$ Sumaira Ameer ${ }^{1} \cdot$ Fouzia Javed $^{1} \cdot$ M. Y. Malik ${ }^{2}$ \\ Received: 31 July 2018 / Accepted: 13 December 2018 / Published online: 5 February 2019 \\ (c) The Author(s) 2019
}

\begin{abstract}
In the present paper, wire coating process using viscoelastic non-Newtonian fluid is investigated along the effects of heat transfer, Joule heating and magnetohydrodynamic fluid flow. Temperature-dependent variable viscosity models are used. The boundary layer equations governing the flow and heat transfer phenomena are solved by applying powerful numerical technique. The notable aspect of the present study is to include porous matrix, which acts as an insulator to prevent heat loss. Similarly, the impact of heat generation is discussed because it controls heat transfer rates. The influence of non-Newtonian parameter, magnetic parameter, permeability parameter, heat generation/absorption parameter, etc. on wire coating is analyzed by graphs.
\end{abstract}

Keywords Non-Newtonian wire coating $\cdot$ Viscoelastic fluid model $\cdot$ Magnetohydrodynamic flow $\cdot$ Heat generation/ absorption $\cdot$ Spongy medium

\section{Introduction}

Many fluids dealt by engineers and scientist, such as air, water and oil can be regarded as Newtonian fluids. However, in many cases, the premise of Newtonian behavior is not rational and rather more complex so non-Newtonian response must be molded. Many fluid materials such as glue, custard, paint, blood and ketchup present non-Newtonian fluid behavior. Due to its wide range of applications in industry, chemical engineering, petroleum engineering, etc., it has gained a lot of importance by many researchers [1-8]. Ellahi et al. [9] studied non-Newtonian micropolar fluid in arterial blood flow through composite stenosis. Among these non-Newtonian fluids, one is Eyring-Powell fluid, it was firstly introduced by Eyring and Powell in 1944. Researchers [10-14] have discussed various aspects of Eyring-Powell fluid.

Technical Editor: Cezar Negrao, PhD.

Azad Hussain

azad.hussain@uog.edu.pk

1 Department of Mathematics, University of Gujrat, Gujrat 50700, India

2 Department of Mathematics, Faculty of Science, King Khalid University, Abha 61413, Saudi Arabia
Wire coating process is very necessary to prevent injuries and reduce the reduction that can be created by machine vibration. In industries, different melt polymers are in use to coat the wire. For wire coating, generally two processes are used. In first, melt polymer is deposited continuously on moving wire, and in second, wire is pulled through the die suffused with viscoelastic material. For wire coating, three different processes are used known as coaxial process, dripping process and electrostatistical deposition process. The dipping process in wire coating process gives much stronger association among the continuums but is slow when compared to other two processes. A typical process of wire coating is demonstrated below in Fig. 1.

It consists of a payoff device, straightener, preheater, extruder device and die, cooling device, capstan, tester and a take-up reel. In this process, the uncoated wire is rolled on the payoff device which passes through straighter, then, temperature is given to the wire through preheater, and a crosshead die contains a canonical die where it assembles the melt polymer and gets coated. After it, this coated wire is cooled by cooling device and then passes along a capstan and a tester, and at the end, coated wire is winded at takeup reel. Many researchers [15-23] investigated wire coating phenomena using different non-Newtonian fluids.

In magnetohydrodynamic, the applied magnetic field produces current due to its Lorentz force, which affects fluid motion impressively. These days, magnetohydrodynamic 


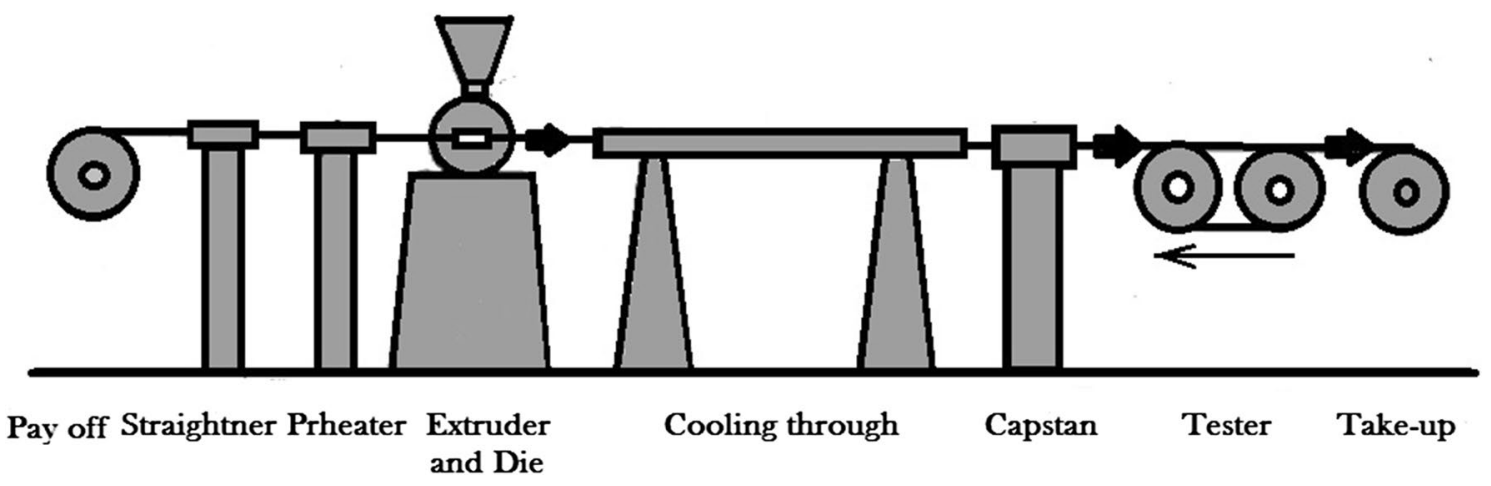

Fig. 1 A typical wire coating process

has become an important topic for research due to its usage at high rate in numerous industrial processes like magnetic field material processing and glass manufacturing. Magnetohydrodynamic treats the electrically conducting fluid flows in the existence of magnetic field. Many researchers [24-30] remit appreciable regard to the study of magnetohydrodynamic flow problems.

Fluid flow in porous media has great importance for researchers due to its wide range of applications in engineering field. Carbonated rocks, wood, metal foams, etc. are various well-known forms of porous media. These days, a very thin porous layer has been used in many industrial and domestic applications such as filters, printing papers, fuel cells and batteries. Many researchers [31-34] also paid a lot of attention to porous media.

The interest in heat transfer of non-Newtonian fluid flows is increasing with the passage of time due to its usage in various industries. Rehman and Nadeem [35] carried out heat transfer analysis for three-dimensional stagnation point flow. Ahmed and many other researchers [36-40] discussed the impact of heat transfer analysis and magnetohydrodynamic fluid.

To the best of authors' knowledge, no one has still studied wire coating process using magnetohydrodynamic flow of viscoelastic Eyring-Powell fluid as coating material.
The objective of the present work is to discuss the process of wire coating with the effects of heat generation and porous media with temperature-dependent variable viscosity using Reynolds and Vogel's model.

\section{Modeling of wire coating}

The geometry of the problem under examination is viewed in Fig. 2. Here $L$ is the length of pressure-type die, $R_{d}$ is the radius and $\theta_{d}$ is the temperature which is saturated by an incompressible elastic-viscous Eyring-Powell fluid. The wire is dragged through center line of die in a stationary pressure-type die when the temperature of wire is indicated with $\theta_{\mathrm{w}}$, radius $R_{\mathrm{w}}$ and velocity $U_{\mathrm{w}}$ in porous medium. Emerging fluid is worked simultaneous by a constant pressure gradient $\frac{\mathrm{d} p}{\mathrm{~d} z}$ parallel to axis of body and a transverse magnetic field with power $B_{o}$. The magnetic field is making right angle with incompressible Eyring-Powell fluid flow's direction. The magnetic Reynolds number is used as minor to ignore the urge magnetic field in our present problem. The die and wire are coaxial. Coordinate system is taken along the axis of the wire.

Fig. 2 Geometry of the problem

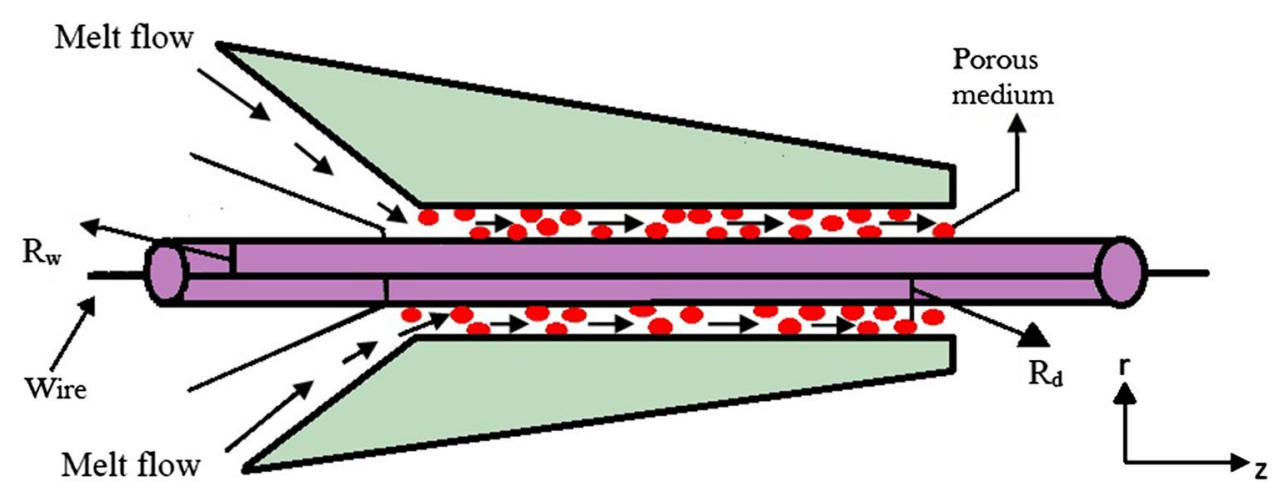


The suitable expressions for velocity of fluid $(\vec{q})$, extra stress tensor $(S)$ and temperature field $(\theta)$ for above-mentioned problem may be considered as

$\overleftrightarrow{\mathbf{q}}=0 \underline{i}+0 j+w(r) \underline{k}$,

$S=S(r)$,

$\theta=\theta(r)$

The Cauchy stress tensor of viscoelastic Eyring-Powell fluid is expressed as

$S=\mu \nabla \mathbf{v}+\frac{1}{\beta} \sinh ^{-1}\left(\frac{1}{C} \nabla \mathbf{v}\right)$,

where $\mu$ is the shear viscosity, $S$ is the Cauchy stress tensor, $C$ is the material constant, $V$ is the velocity and $C$ is the material constant. Equation (4) is simplified as

$\sinh ^{-1}\left(\frac{1}{C} \nabla \mathbf{v}\right) \approx \frac{1}{C} \nabla \mathbf{v}-\frac{1}{6}\left(\frac{1}{C} \nabla \mathbf{v}\right)^{3}, \quad\left|\frac{1}{C} \nabla \mathbf{v}\right| \ll 1$.

The suitable boundary conditions for the present consideration can be defined as

$w\left(R_{\mathrm{w}}\right)=U_{\mathrm{w}}, \quad \theta\left(R_{\mathrm{w}}\right)=\theta_{\mathrm{w}}$,

$w\left(R_{d}\right)=0, \quad \theta\left(R_{d}\right)=\theta_{d}$.

The governing equations are

$\overleftrightarrow{\nabla} \cdot \vec{q}=0$

$\rho\left(\frac{D \overleftrightarrow{q}}{D t}\right)=\overleftrightarrow{F}-\overleftrightarrow{\nabla} p+\overleftrightarrow{J} \times \overleftrightarrow{B}+\frac{\mu \overleftrightarrow{q}}{K_{\mathrm{p}}^{*}}$

$\rho C_{p} \frac{D \theta}{D t}=k \nabla^{2}+\varphi+Q_{0}\left(\theta-\theta_{\mathrm{w}}\right)+J_{\mathrm{d}}$,

where $\vec{q}$ is velocity vector, $\rho$ represent density, $\frac{D}{D t}$ is temporal derivative, $\overleftrightarrow{J} \times \overleftrightarrow{B}$ indicates electromagnetic origin per unit volume appears due to the correspondence of magnetic arena, current $Q_{0}$ represents the rate of volumetric heat generation and $J_{\mathrm{d}}$ is the Joule dissipation term. The magnetic body force produced along the $z$-direction can be defined as

$\overleftrightarrow{J} \times \overleftrightarrow{B}=\left(0,0, \sigma \beta_{0}^{2} w\right)$

Applying (1-3), the continuity of Eq. (7) is identically satisfied and we get nonvanishing components of extra stress tensor $S$ as

$S_{z r}=\left(\mu+\frac{1}{\beta C}\right) \frac{\mathrm{d} w}{\mathrm{~d} r}-\frac{1}{6 \beta C^{3}}\left(\frac{\mathrm{d} w}{\mathrm{~d} r}\right)^{3}$.

Putting the velocity field and Eqs. (10-11) in Eq. (8), we get $\frac{\partial P}{\partial r}=0$,

$\frac{\partial P}{\partial \theta}=0$,

$\frac{\partial P}{\partial z}=\frac{1}{r} \frac{\mathrm{d}}{\mathrm{d} r}\left[r\left\{\left(\mu+\frac{1}{\beta C}\right) \frac{\mathrm{d} w}{\mathrm{~d} r}-\frac{1}{6 \beta C}\left(\frac{\mathrm{d} w}{\mathrm{~d} r}\right)^{3}\right\}\right]-\sigma \beta_{0}^{2} w-\frac{\mu w}{K_{\mathrm{p}}^{*}}$.

However, Eq. (14) shows the flow owing the pressure gradient. When we leave the die then, only drag of wire happened. That's why pressure gradient is contributing nothing in the axial direction. So Eq. (14) takes the form as

$\frac{1}{r} \frac{\mathrm{d}}{\mathrm{d} r}\left[r\left\{\left(\mu+\frac{1}{\beta C}\right) \frac{\mathrm{d} w}{\mathrm{~d} r}-\frac{1}{6 \beta C}\left(\frac{\mathrm{d} w}{\mathrm{~d} r}\right)^{3}\right\}\right]-\sigma \beta_{0}^{2} w-\frac{\mu w}{K_{\mathrm{p}}^{*}}=0$,

and energy Eq. (9) becomes

$K\left(\frac{\mathrm{d}^{2} \theta}{\mathrm{d} r^{2}}+\frac{1}{r} \frac{\mathrm{d} \theta}{\mathrm{d} r}\right)$

$+\left(\left(\mu+\frac{1}{\beta C}\right) \frac{\mathrm{d} w}{\mathrm{~d} r}-\frac{1}{6 \beta C^{3}}\left(\frac{\mathrm{d} w}{\mathrm{~d} r}\right)^{3}\right)$.

$\frac{\mathrm{d} w}{\mathrm{~d} r}+Q_{0}\left(\theta-\theta_{\mathrm{w}}\right)+\sigma \beta_{0}^{2} w^{2}=0$.

\section{Constant viscosity}

Defining dimensionless parameters as

$r^{*}=\frac{r}{R_{\mathrm{w}}}, \quad w^{*}=\frac{w}{U_{\mathrm{w}}}, \quad M^{2}=\frac{\sigma \beta_{0}^{2} R_{\mathrm{w}}^{2}}{\mu}$,

$K_{\mathrm{p}}=\frac{R_{\mathrm{w}}^{2}}{K_{\mathrm{p}}^{*}}, \quad w=\frac{v_{0}}{U_{\mathrm{w}}}, \quad N=\frac{1}{\mu \beta C}$,

$\theta^{*}=\frac{\left(\theta-\theta_{\mathrm{w}}\right)}{\left(\theta_{d}-\theta_{\mathrm{w}}\right)}, \quad Q=\frac{Q_{0} R_{\mathrm{w}}^{2}}{K}$,

$B_{r}=\frac{\mu U_{\mathrm{w}}^{2}}{K\left(\theta_{d}-\theta_{\mathrm{w}}\right)}, \quad R_{\mathrm{w}}=\frac{\beta v_{0}}{\mu}, \quad \varepsilon=\frac{\mu}{6 w^{2}(\beta C)^{3}}$.

Using these new variables in Eqs. (15) and (16) with Eq. (6) and after removing asterisks, we get the following form

$$
\begin{aligned}
& \begin{aligned}
(1+N)[ & {\left[r \frac{\mathrm{d}^{2} w}{\mathrm{~d} r^{2}}+\frac{\mathrm{d} w}{\mathrm{~d} r}\right]-\varepsilon\left[\left(\frac{\mathrm{d} w}{\mathrm{~d} r}\right)^{3}+3 r\left(\frac{\mathrm{d} w}{\mathrm{~d} r}\right)^{2} \frac{\mathrm{d}^{2} w}{\mathrm{~d} r^{2}}\right] } \\
& -M^{2} w r-K_{\mathrm{p}} w r=0
\end{aligned} \\
& \begin{aligned}
& w(1)= 1 \quad \text { and } \quad w(\delta)=0 . \\
& \frac{\mathrm{d}^{2} \theta}{\mathrm{d} r^{2}}+\frac{1}{r} \frac{\mathrm{d} \theta}{\mathrm{d} r}+B_{r}(1+N)\left(\frac{\mathrm{d} w}{\mathrm{~d} r}\right)^{2}+\varepsilon B_{r}\left(\frac{\mathrm{d} w}{\mathrm{~d} r}\right)^{4}+Q \theta+B_{r} M^{2} w^{2}=0
\end{aligned}
\end{aligned}
$$

$\theta(1)=0 \quad$ and $\quad \theta(\delta)=1$. 


\section{Reynolds model}

Here, we used Reynolds model to explain temperaturedependent viscosity. The dimensionless viscosity can be expressed for Reynolds model as

$\mu \approx 1-\beta_{0} m \theta$.

It will be applied for variation of temperature-dependent viscosity, while $m$ is used for viscosity parameter. Using nondimensional parameters,

$r^{*}=\frac{r}{R_{\mathrm{w}}}, \quad w^{*}=\frac{w}{U_{\mathrm{w}}}, \quad M^{2}=\frac{\sigma \beta_{0}^{2} R_{\mathrm{w}}^{2}}{\mu_{0}}$,

$K_{\mathrm{p}}=\frac{R_{\mathrm{w}}^{2}}{K_{\mathrm{p}}^{*}}, \quad w=\frac{v_{0}}{U_{\mathrm{w}}}, \quad N=\frac{1}{\mu_{0} \beta C}, \quad \mu^{*}=\frac{\mu}{\mu_{0}}$,

$\theta^{*}=\frac{\left(\theta-\theta_{\mathrm{w}}\right)}{\left(\theta_{d}-\theta_{\mathrm{w}}\right)}, \quad Q=\frac{Q_{0} R_{\mathrm{w}}^{2}}{K}$,

$B_{r}=\frac{\mu_{0} U_{\mathrm{w}}^{2}}{K\left(\theta_{d}-\theta_{\mathrm{w}}\right)}, \quad R_{\mathrm{w}}=\frac{\beta v_{0}}{\mu_{0}}, \quad \varepsilon=\frac{\mu_{0}}{6 w^{2}(\beta C)^{3}}$.

After removing asterisks, we obtain nondimensional form of momentum and energy equation along boundary conditions

$$
\begin{aligned}
& \frac{\mathrm{d}^{2} w}{\mathrm{~d} r^{2}}\left[r\left(1-\beta_{0} m \theta\right)+r N-3 r \varepsilon\left(\frac{\mathrm{d} w}{\mathrm{~d} r}\right)^{2}\right] \\
& +\frac{\mathrm{d} w}{\mathrm{~d} r}\left[1-\beta_{0} m \theta+N-\beta_{0} m r \frac{\mathrm{d} \theta}{\mathrm{d} r}\right]-\varepsilon\left(\frac{\mathrm{d} w}{\mathrm{~d} r}\right)^{3} \\
& -K_{\mathrm{p}} w r-M^{2} w r=0, \\
& w(1)=1 \quad \text { and } \quad w(\delta)=0, \\
& \text { and } \\
& \frac{\mathrm{d}^{2} \theta}{\mathrm{d} r^{2}}+\frac{1}{r} \frac{\mathrm{d} \theta}{\mathrm{d} r}+\left(1-\beta_{0} m \theta\right) B_{r}\left(\frac{\mathrm{d} w}{\mathrm{~d} r}\right)^{2} \\
& +B_{r}\left(\frac{\mathrm{d} w}{\mathrm{~d} r}\right)^{2}(N+\varepsilon)+Q \theta+B_{r} M^{2} w^{2}=0, \\
& \theta(1)=0 \quad \text { and } \quad \theta(\delta)=1 .
\end{aligned}
$$

\section{Vogel's model}

In this case, we take temperature-dependent viscosity as

$\mu=\mu_{0} \exp \left(\frac{D}{B^{\prime}+\theta}-\theta_{\mathrm{w}}\right)$.

Applying expansions, we get

$\mu=\Omega\left(1-\frac{D}{B^{\prime 2}} \theta\right)$,

where $D, B$ are parameters of viscosity and $\Omega=\mu_{0} \exp \left(\frac{D}{B^{\prime 2}}-\theta_{\mathrm{w}}\right)$.
We obtain nondimensional equations of momentum and energy along boundary conditions after removing asterisks

$$
\begin{gathered}
\frac{\mathrm{d}^{2} w}{\mathrm{~d} r^{2}}\left[r \Omega\left(1-\frac{D}{B^{\prime 2}} \theta\right)+r N-3 r \varepsilon\left(\frac{\mathrm{d} w}{\mathrm{~d} r}\right)^{2}\right] \\
+\frac{\mathrm{d} w}{\mathrm{~d} r}\left[\Omega\left(1-\frac{D}{B^{\prime 2}} \theta\right)+N-\Omega \frac{D}{B^{\prime 2}} r \frac{\mathrm{d} \theta}{\mathrm{d} r}\right] \\
-\varepsilon\left(\frac{\mathrm{d} w}{\mathrm{~d} r}\right)^{3}-K_{p} w r-M^{2} w r=0,
\end{gathered}
$$

$w(1)=1 \quad$ and $\quad w(\delta)=0$,

and

$\frac{\mathrm{d}^{2} \theta}{\mathrm{d} r^{2}}+\frac{1}{r} \frac{\mathrm{d} \theta}{\mathrm{d} r}+\Omega\left(1-\frac{D}{B^{\prime 2}} \theta\right) B_{r}\left(\frac{\mathrm{d} w}{\mathrm{~d} r}\right)^{2}$

$+B_{r}\left(\frac{\mathrm{d} w}{\mathrm{~d} r}\right)^{2}(N+\varepsilon)+Q \theta+B_{r} M^{2} w^{2}=0$,

$\theta(1)=0 \quad$ and $\quad \theta(\delta)=1$.

\section{Numerical solution}

\subsection{Constant viscosity}

The governing higher-order differential equations are firstly converted into first-order ordinary differential equations. They are solved numerically utilizing Runge-Kutta method with shooting technique. First of all, we convert momentum and energy equation into first-order form. Equations (18) and (20) become

$$
\frac{\mathrm{d}^{2} w}{\mathrm{~d} r^{2}}=\frac{\varepsilon\left(\frac{\mathrm{d} w}{\mathrm{~d} r}\right)^{3}-(1+N) \frac{\mathrm{d} w}{\mathrm{~d} r}+M^{2} w r+K_{p} w r}{(1+N) r+3 r \varepsilon\left(\frac{\mathrm{d} w}{\mathrm{~d} r}\right)^{2}}
$$

$$
\frac{\mathrm{d}^{2} \theta}{\mathrm{d} r^{2}}=-\left[\frac{1}{r} \frac{\mathrm{d} \theta}{\mathrm{d} r}+B_{r}(1+N)\left(\frac{\mathrm{d} w}{\mathrm{~d} r}\right)^{2}+\varepsilon B_{r}\left(\frac{\mathrm{d} w}{\mathrm{~d} r}\right)^{4}+Q \theta+B_{r} M^{2} w^{2}\right] .
$$

Defining new variables to convert higher-order ordinary differential equation into first order as

$w=y_{1}, \quad w^{\prime}=y_{2}, \quad w^{\prime \prime}=y_{2}^{\prime}, \quad \theta=y_{3}, \quad \theta^{\prime}=y_{4}, \quad \theta^{\prime \prime}=y_{4}^{\prime}$,

$y_{2}^{\prime}=\frac{\varepsilon\left(y_{2}\right)^{3}-(1+N) y_{2}+M^{2} y_{1} r+K_{p} y_{1} r}{(1+N) r+3 r \varepsilon\left(y_{2}\right)^{2}}$,

$y_{4}^{\prime}=-\left[\frac{1}{r} y_{4}+B_{r}(1+N)\left(y_{2}\right)^{2}+\varepsilon B_{r}\left(y_{2}\right)^{4}+Q y_{3}+B_{r} M^{2} y_{1}^{2}\right]$.

The boundary conditions convert into initial conditions as

$y_{1}(1)=1 \quad$ and $\quad y_{1}(\delta)=0$, 
$y_{3}(1)=0 \quad$ and $\quad y_{3}(\delta)=1$.

\subsection{Reynolds model}

Equations (24) and (26) may be written as

$\frac{\mathrm{d}^{2} w}{\mathrm{~d} r^{2}}=\frac{\varepsilon\left(\frac{\mathrm{d} w}{\mathrm{~d} r}\right)^{3}+K_{p} w r+M^{2} w r-\frac{\mathrm{d} w}{\mathrm{~d} r}\left[1-\beta_{0} m \theta+N-\beta_{0} m r \frac{\mathrm{d} \theta}{\mathrm{d} r}\right]}{\left[r\left(1-\beta_{0} m \theta\right)+r N-3 r \varepsilon\left(\frac{\mathrm{d} w}{\mathrm{~d} r}\right)^{2}\right]}$,

$$
\begin{aligned}
\frac{\mathrm{d}^{2} \theta}{\mathrm{d} r^{2}}= & -\left[\frac{1}{r} \frac{\mathrm{d} \theta}{\mathrm{d} r}+\left(1-\beta_{0} m \theta\right) B_{r}\left(\frac{\mathrm{d} w}{\mathrm{~d} r}\right)^{2}\right. \\
& \left.+B_{r}\left(\frac{\mathrm{d} w}{\mathrm{~d} r}\right)^{2}(N+\varepsilon)+Q \theta+B_{r} M^{2} w^{2}\right] .
\end{aligned}
$$

Using variables from Eq. (36) to reduce higher-order differential equation into first order as

$$
y_{2}^{\prime}=\frac{\varepsilon\left(y_{2}\right)^{3}+K_{p} y_{1} r+M^{2} y_{1} r-y_{2}\left[1-\beta_{0} m y_{3}+N-\beta_{0} m r y_{4}\right]}{\left[r\left(1-\beta_{0} m y_{3}\right)+r N-3 r \varepsilon\left(y_{2}\right)^{2}\right]},
$$

$$
\begin{aligned}
y_{4}^{\prime}=- & {\left[\frac{1}{r} y_{4}+\left(1-\beta_{0} m y_{3}\right) B_{r}\left(y_{2}\right)^{2}\right.} \\
& \left.+B_{r}\left(y_{2}\right)^{2}(N+\varepsilon)+Q y_{3}+B_{r} M^{2} y_{1}^{2}\right] .
\end{aligned}
$$

The boundary conditions converted into initial conditions as

$$
\begin{array}{ll}
y_{1}(1)=1 \quad \text { and } \quad y_{1}(\delta)=0, \\
y_{3}(1)=0 \quad \text { and } \quad y_{3}(\delta)=1 .
\end{array}
$$

\subsection{Vogel's model}

Equations (30) and (32) can be written as

$$
\begin{aligned}
\frac{\mathrm{d}^{2} w}{\mathrm{~d} r^{2}}= & \frac{\varepsilon\left(\frac{\mathrm{d} w}{\mathrm{~d} r}\right)^{3}+K_{p} w r+M^{2} w r-\frac{\mathrm{d} w}{\mathrm{~d} r}\left[\Omega\left(1-\frac{D}{B^{\prime 2}} \theta\right)+N-\Omega \frac{D}{B^{\prime 2}} r \frac{\mathrm{d} \theta}{\mathrm{d} r}\right]}{r \Omega\left(1-\frac{D}{B^{\prime 2}} \theta\right)+r N-3 r \varepsilon\left(\frac{\mathrm{d} w}{\mathrm{~d} r}\right)^{2}} \\
\frac{\mathrm{d}^{2} \theta}{\mathrm{d} r^{2}}= & -\left[\frac{1}{r} \frac{\mathrm{d} \theta}{\mathrm{d} r}+\Omega\left(1-\frac{D}{B^{\prime 2}} \theta\right) B_{r}\left(\frac{\mathrm{d} w}{\mathrm{~d} r}\right)^{2}\right. \\
& \left.+B_{r}\left(\frac{\mathrm{d} w}{\mathrm{~d} r}\right)^{2}(N+\varepsilon)+Q \theta+B_{r} M^{2} w^{2}\right]
\end{aligned}
$$

Using Eq. (36) in Eqs. (47) and (48), we get

$$
y_{2}^{\prime}=\frac{\varepsilon\left(y_{2}\right)^{3}+K_{p} y_{1} r+M^{2} y_{1} r-y_{2}\left[\Omega\left(1-\frac{D}{B^{\prime 2}} y_{3}\right)+N-\Omega \frac{D}{B^{\prime 2}} r y_{4}\right]}{r \Omega\left(1-\frac{D}{B^{\prime 2}} y_{3}\right)+r N-3 r \varepsilon\left(y_{2}\right)^{2}},
$$

$$
\begin{aligned}
y_{4}^{\prime}=- & {\left[\frac{1}{r} y_{4}+\Omega\left(1-\frac{D}{B^{\prime 2}} y_{3}\right) B_{r}\left(y_{2}\right)^{2}\right.} \\
& \left.+B_{r}\left(y_{2}\right)^{2}(N+\varepsilon)+Q y_{3}+B_{r} M^{2} y_{1}^{2}\right] .
\end{aligned}
$$

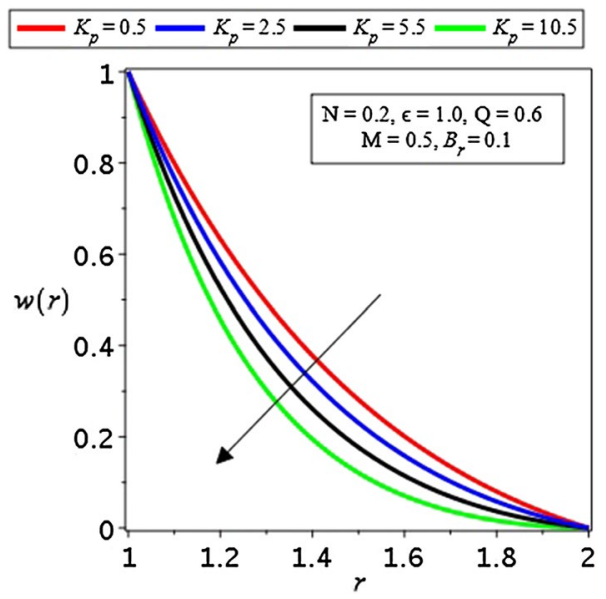

Fig. 3 Effects of $K_{\mathrm{p}}$ on velocity distribution

Along boundary conditions

$y_{1}(1)=1 \quad$ and $\quad y_{1}(\delta)=0$,

$y_{3}(1)=0 \quad$ and $\quad y_{3}(\delta)=1$.

\section{Graphical results and discussions}

In this work, we examine Eyring-Powell fluid as coating material for wire. The process of wire coating is occurring in a die with uniform magnetic and heat generation effects in porous medium. The effects of different emerging physical parameters known as non-Newtonian parameter $\beta_{0}$, heat generation parameter $Q$, viscosity parameters $m$ and $\Omega$ for Reynolds and Vogel's models, respectively, porous parameter $K_{\mathrm{p}}$, Brinkman number $B_{r}$ and other parameters $D$ and $M$ on velocity and temperature profile are expressed by graphs. Figure 2 displays geometry of given problem. Figure 3 presents the result of $K_{\mathrm{p}}$ over velocity profile for constant viscosity when $B_{r}, K_{\mathrm{p}}$ and $Q$ remain constant. The velocity profile decreased by enlargement in the worth of $K_{\mathrm{p}}$. Figure 4 proposes the ascendancy of $\varepsilon$ on velocity profile when viscosity is constant and having other parameters as constant. The velocity profile presents increasing behavior because of escalating $\varepsilon$. Figure 5 shows the effect of $M$ on velocity profile. Figure 6 points out the $B_{r}$ on velocity profile for Reynolds model. Velocity profile shows increasing actions owing to escalating $B_{r}$. Figure 7 interprets the outcomes of permeability parameter on velocity profile for Reynolds model when $\beta_{0}=0.1, M=0.6, B_{r}=0.1, Q=0.1$ and $m=0.3$. Figure 8 illuminates the influence of $N$ on velocity profile for Reynolds model. Velocity curve eliminates the increasing action due to increase in $N$. Figure 9 expounds that velocity profile shows increasing response by 


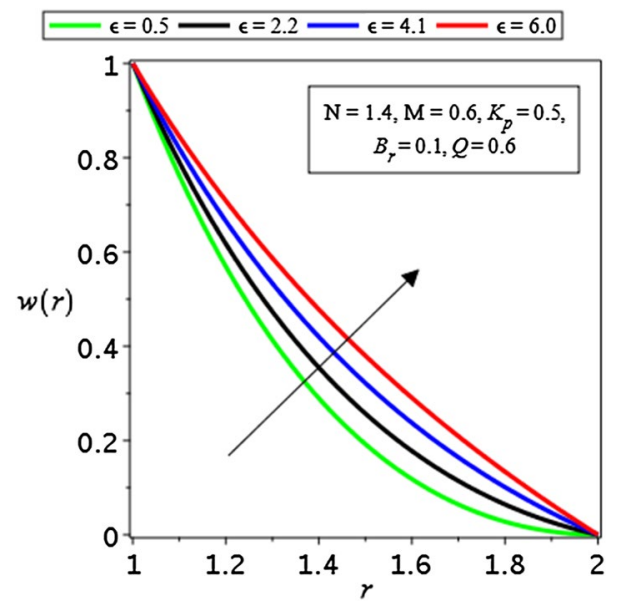

Fig. 4 Influence of $\epsilon$ on velocity profile

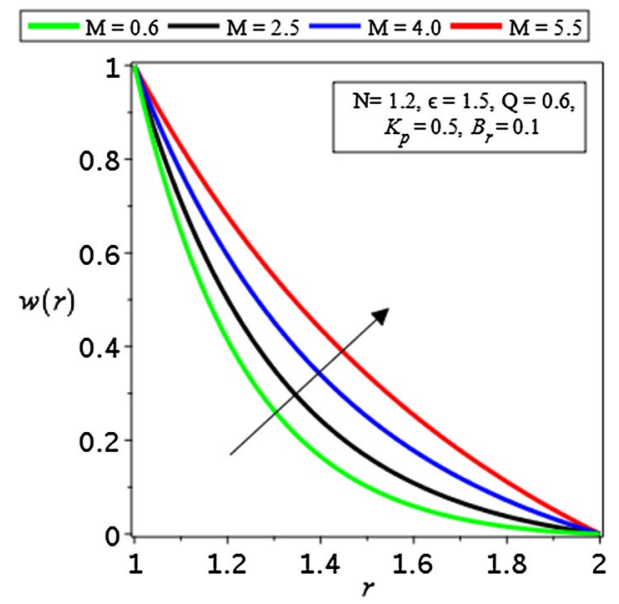

Fig. 5 Influence of $M$ on velocity distribution

accelerating $B_{r}$ for Vogel's model, while $M=0.11, K_{\mathrm{p}}=0.1$ and $Q=0.2$. Figure 10 comes out that the velocity distribution illustrates increasing actions by accelerating $D$ for Vogel's model. The curve of the graph shows increasing behavior. Figure 11 represents the inclination in velocity profile due to increasing $Q$ for Vogel's model keeping $D=0.2, M=0.11$ and $B_{r}=0.2$. Figure 12 explains the variations in temperature profile resulting due to $\varepsilon$ for constant viscosity when $M=0.6, K_{\mathrm{p}}=0.6$ and $N=0.01$. Velocity profile is downward due to rise in $\varepsilon$. Figure 13 explicates the result of $B_{r}$ coefficient on temperature profile for constant viscosity. Velocity profile is decreasing by accelerating $B_{r}$. Figure 14 presents the effects of $Q$ on temperature profile when viscosity is constant and having other parameters as constant. The velocity profile presents increasing behavior because of escalating $Q$. Figure 15 displays the increasing response of temperature profile due to the boosting in

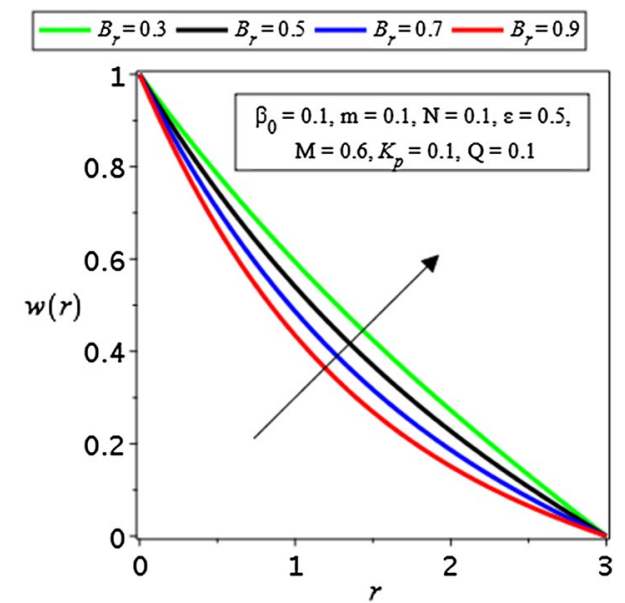

Fig. 6 Effects of $B_{r}$ on velocity distribution in case of Reynolds model

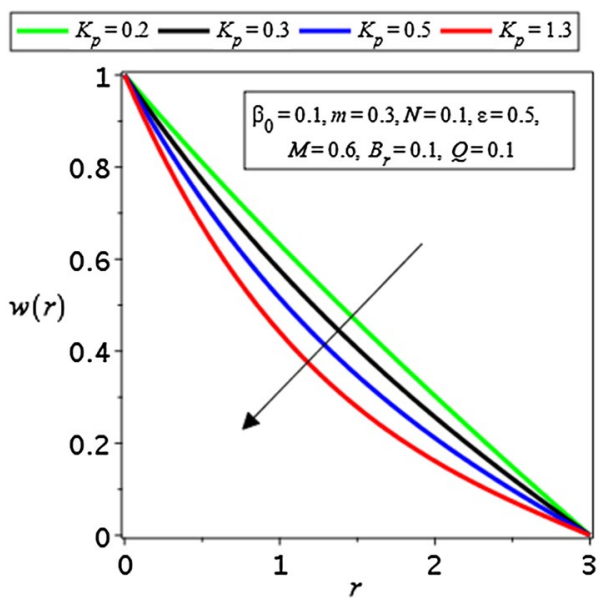

Fig. 7 Impact of $K_{\mathrm{p}}$ on velocity distribution in Reynolds model

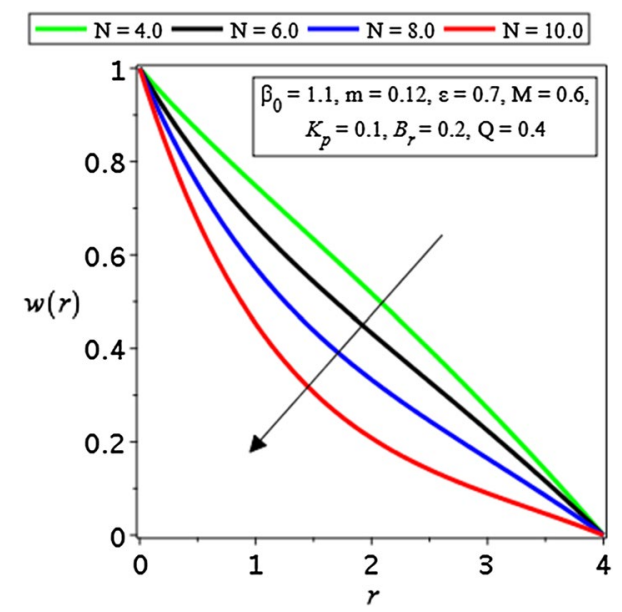

Fig. 8 Effects of $N$ on velocity distribution in Reynolds model 


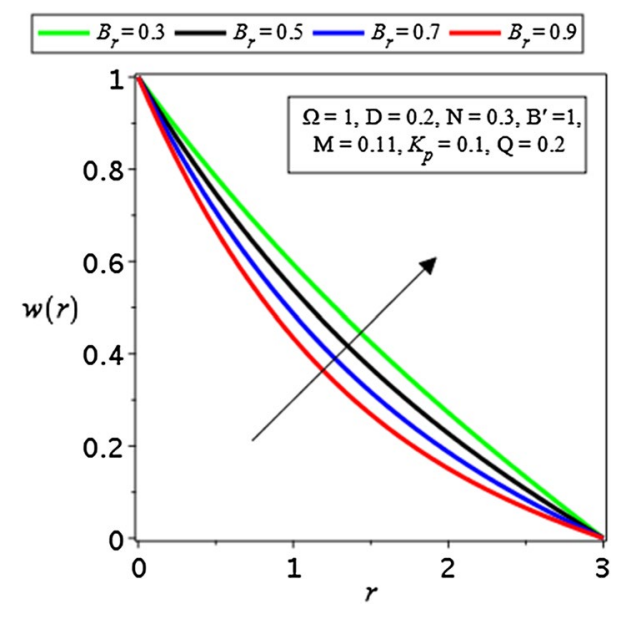

Fig. 9 Effects of $B_{r}$ on velocity distribution in case of Vogel's model

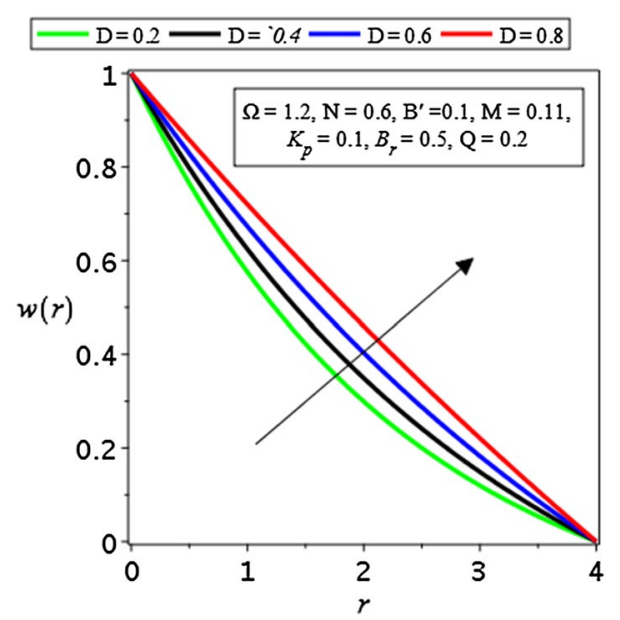

Fig. 10 Impact of $D$ on velocity distribution in case of Vogel's model

value of $\varepsilon$ for Reynolds model. Figure 16 illustrates that temperature distribution goes upward due to increase in $M$ for Reynolds model. The temperature distribution illustrates increasing actions by accelerating $M$ for Reynolds model. Figure 17 comes out that the enlargement in the value of $Q$ curve shows decreasing behavior. Figure 18 expresses that temperature distribution accelerates due to amplification in the value of $M$ for Vogel's model with $D=0.2, K_{\mathrm{p}}=0.1$ and $Q=0.6$. Figure 19 indicates the decreasing temperature curve is caused by increasing $\Omega$ for Vogel's model with $N=0.2, B^{\prime}=1.3, K_{\mathrm{p}}=0.1$ and $D=0.3$. Figure 20 clarifies the S.T lines impact for different worth of $B_{r}$ for constant viscosity. Figure 21 illustrates the effects of stream lines (S.T lines) for distinct values of $B_{r}$ for Reynolds model. Figure 22 clarifies the influence of stream lines on disparate worth of $B_{r}$ for Vogel's model. 3D result for distinct value of $B_{r}$ for constant viscosity is shown in Fig. 23 properly.

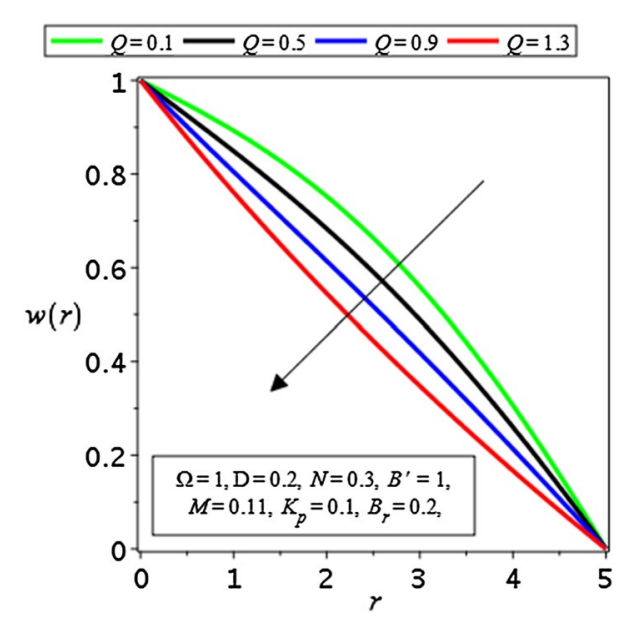

Fig. 11 Impact of $Q$ on velocity distribution for Vogel's model

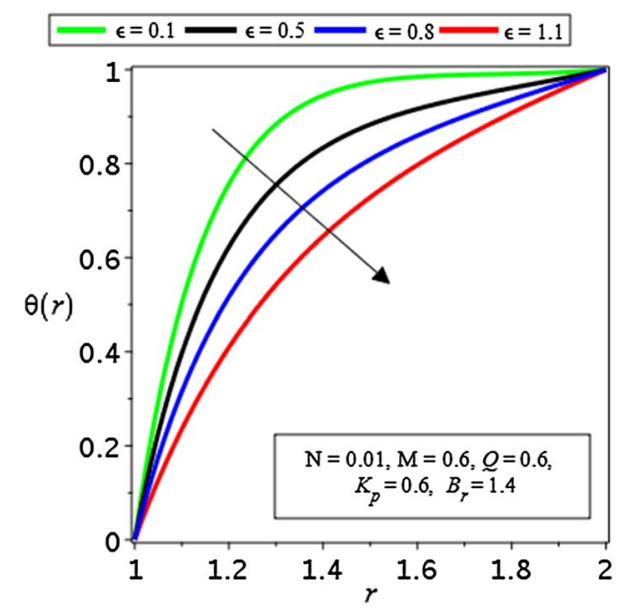

Fig. 12 Influence of $\epsilon$ on temperature distribution

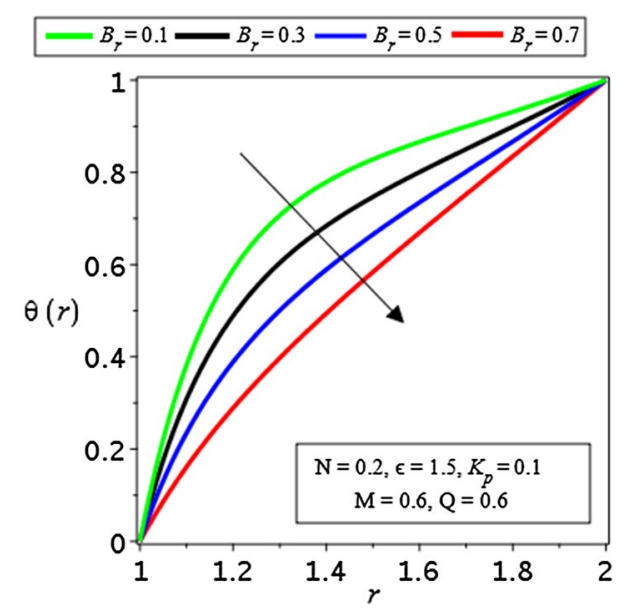

Fig. 13 Impact of $B_{r}$ on temperature distribution 


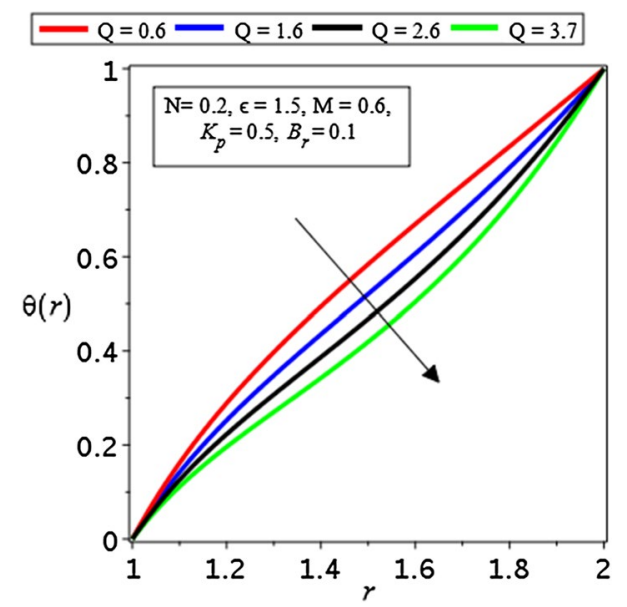

Fig. 14 Influence of $Q$ on temperature profile

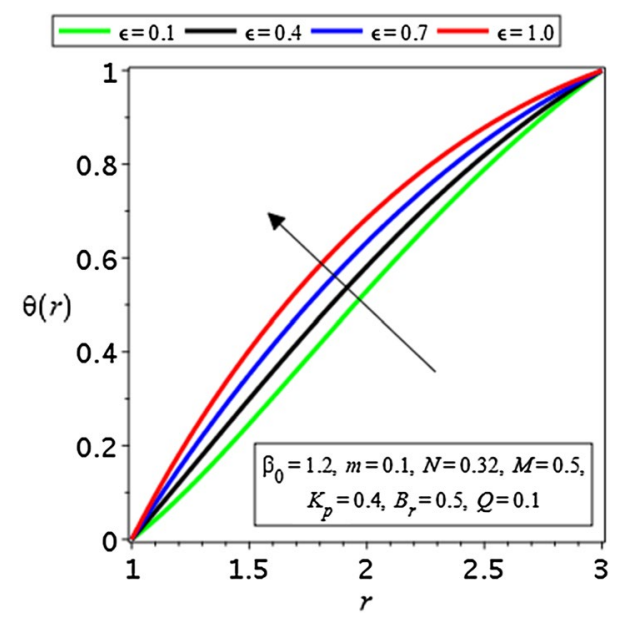

Fig. 15 Influence of $\epsilon$ on temperature distribution for Reynolds model

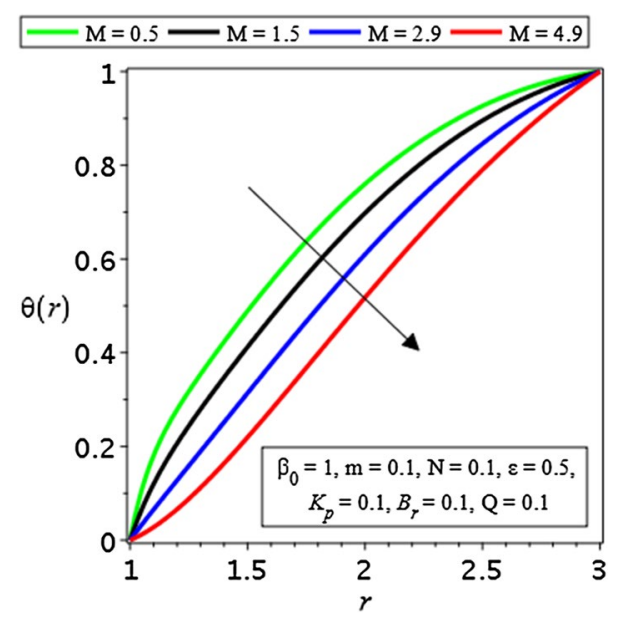

Fig. 16 Influence of $M$ on temperature distribution for Reynolds model

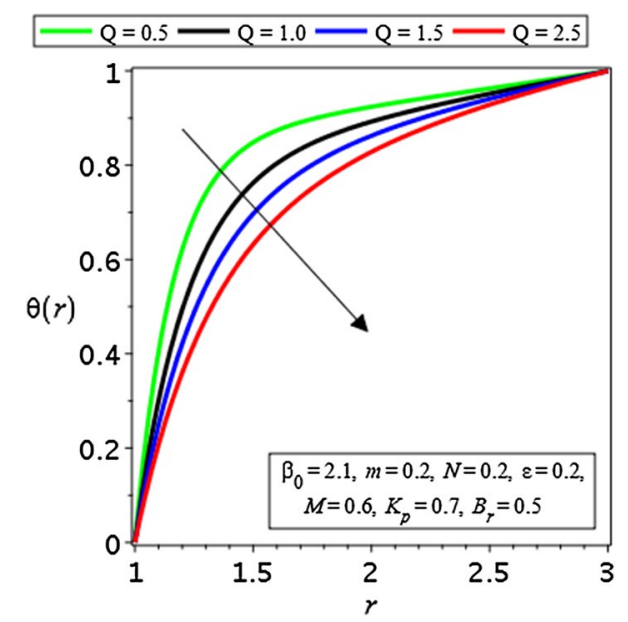

Fig. 17 Impact of $Q$ on temperature distribution in case of Reynolds model

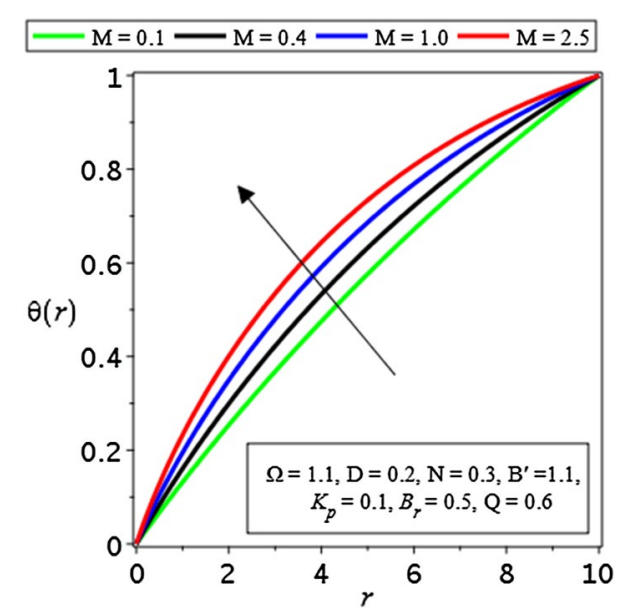

Fig. 18 Effects of $M$ on temperature distribution in case of Vogel's model

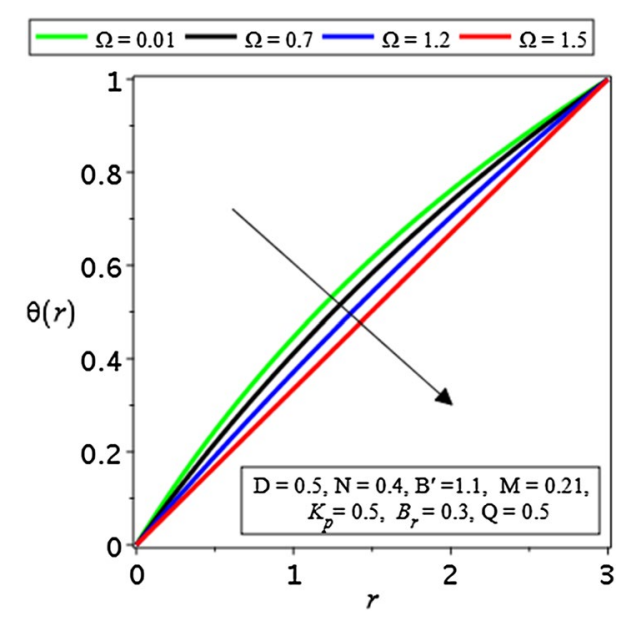

Fig. 19 Impact of $Q$ on temperature distribution for Vogel's model 


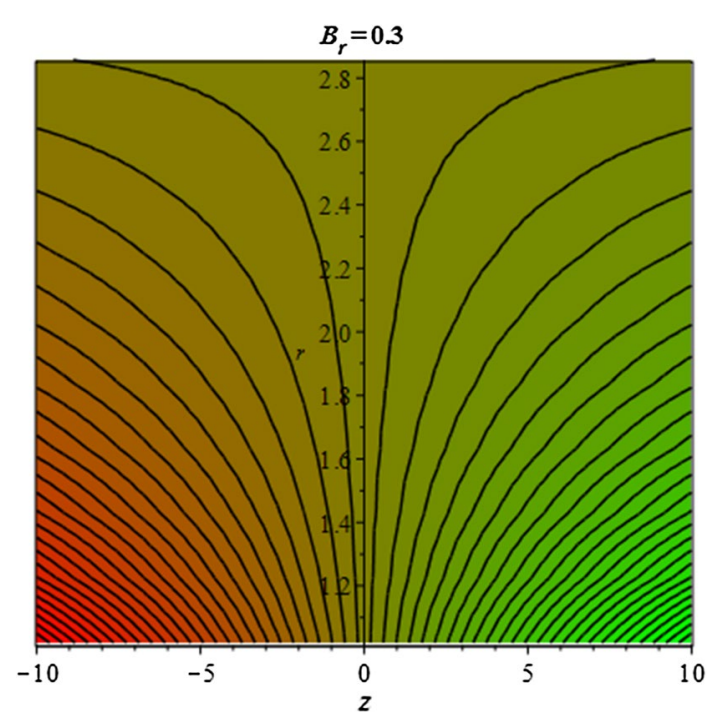

Fig. 20 Stream lines for $B_{r}=0.3$

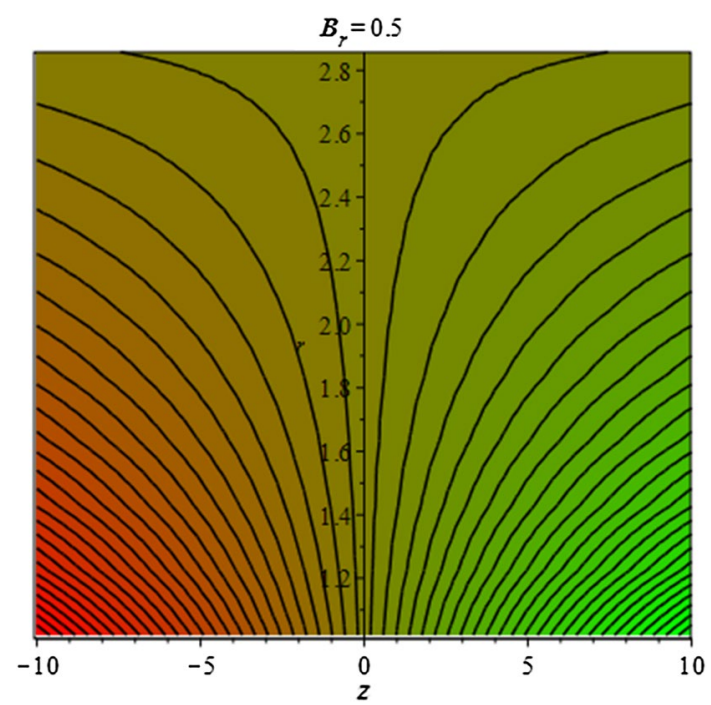

Fig. 21 Stream lines for $B_{r}=0.5$ in case of Reynolds model

Figure 24 shows the 3D impact for distinct value for Reynolds model. Figure 25 expounds the $3 \mathrm{D}$ effects for distinct value of $Q$ for Vogel's model.

\section{Concluding remarks}

In the present work, we have computed impact of magnetohydrodynamic flow and heat transfer in wire coating process using melt polymer in a porous medium along Joule heating and variable viscosity. Wire is coated in a pressure-type die where it meets Eyring-Powell fluid. Porous matrix is used as

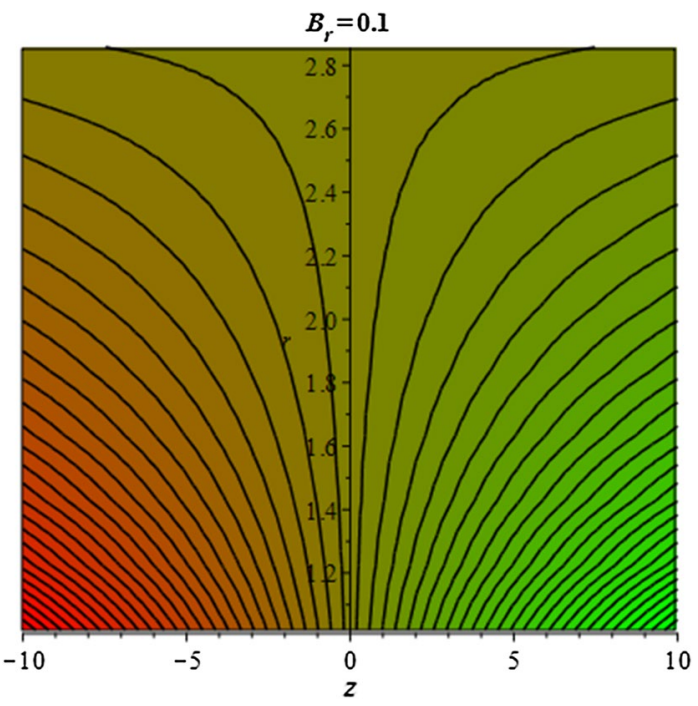

Fig. 22 Stream lines for $B_{r}=0.1$ in case of Vogel's model

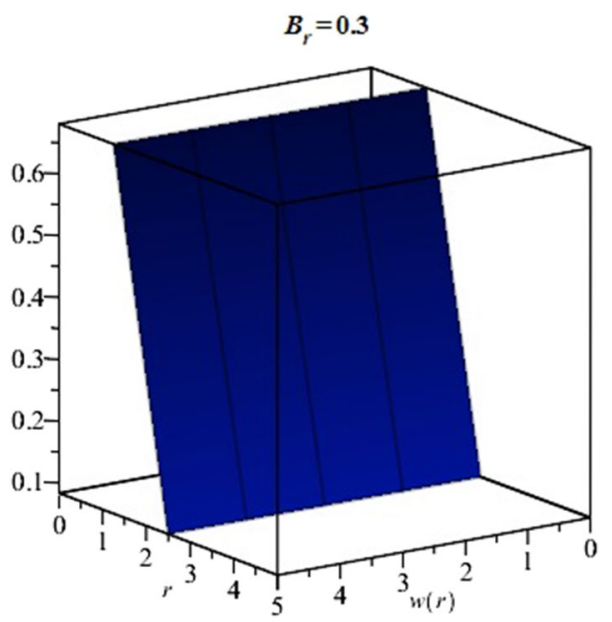

Fig. 23 3-D graph of $w(r)$ for $B_{r}=0.3$

insulator due to which the flow and heat mobility process saves loss of heat and increases the cooling/heating process. The solution of given problem is obtained using shooting method. The result of engaged parameter is presented on velocity profile and temperature distribution. Important points of the current study that are procured are presented below as:

1. The velocity of fluid shows upward behavior by increase in the value of $\varepsilon, M, B_{r}, N$ and $D$ and presents decreasing behavior due to increase in value of $K_{\mathrm{p}}, Q$ and $\varepsilon$.

2. The temperature profile shows flourishing behavior for blowing up in the value of $\varepsilon$ and $M$ and decreasing behavior for the value $B_{r}, Q$ and $\varepsilon$. 


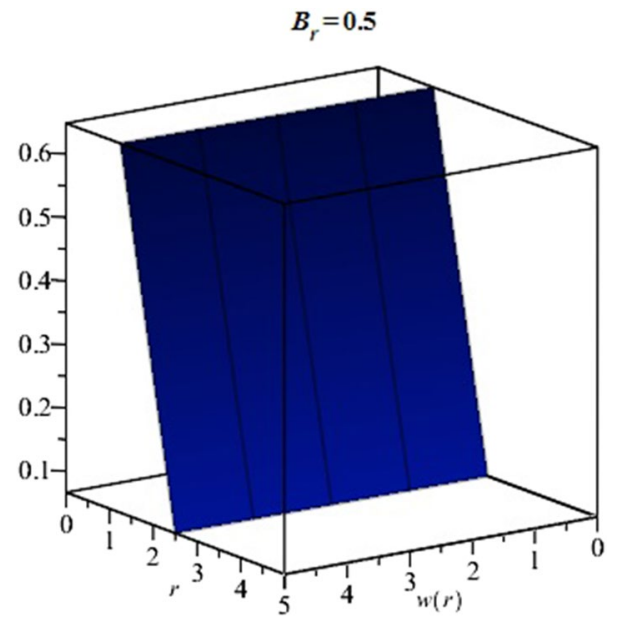

Fig. 24 3-D graph of $w(r)$ for $B_{r}=0.5$ in case of Reynolds model

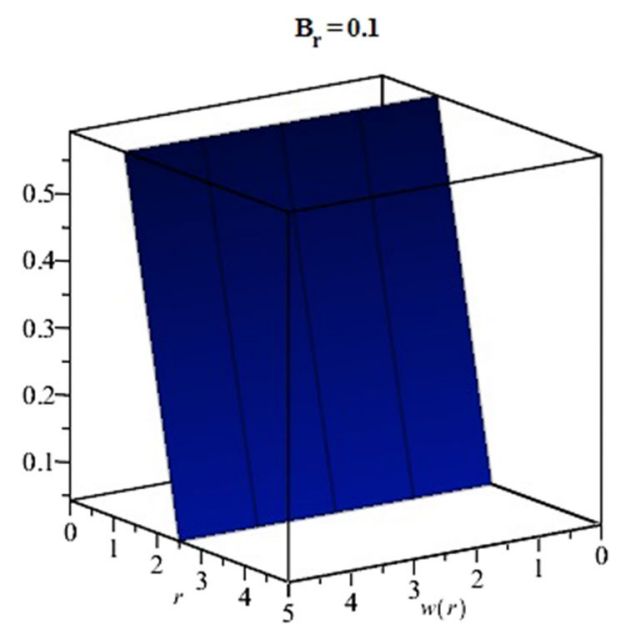

Fig. 25 3-D graph of $w(r)$ for $B_{r}=0.1$ in case of Vogel's model

Open Access This article is distributed under the terms of the Creative Commons Attribution 4.0 International License (http://creativeco mmons.org/licenses/by/4.0/), which permits unrestricted use, distribution, and reproduction in any medium, provided you give appropriate credit to the original author(s) and the source, provide a link to the Creative Commons license, and indicate if changes were made.

\section{References}

1. Nadeem S, Ahmad S, Muhammad N (2017) Cattaneo-Christov flux in the flow of a viscoelastic fluid in the presence of Newtonian heating. J Mol Liq 237:180-184

2. Ijaz S, Iqbal Z, Maraj EN, Nadeem S (2018) Investigation of Cu$\mathrm{CuO} /$ blood mediated transportation in stenosed artery with unique features for theoretical outcomes of hemodynamics. J Mol Liq 254:421-432

3. Abbas N, Saleem S, Nadeem S, Alderremy AA, Khan AU (2018) On stagnation point flow of a micro polar nanofluid past a circular cylinder with velocity and thermal slip. Results Phys 9:1224-1232
4. Ijaz S, Nadeem S (2018) Transportation of nanoparticles investigation as a drug agent to attenuate the atherosclerotic lesion under the wall properties impact Chaos. Solitons Fractals 112:52-65

5. Tabassum R, Mehmood R, Nadeem S (2017) Impact of viscosity variation and micro rotation on oblique transport of $\mathrm{Cu}$-water fluid. J Colloid Interface Sci 501:304-310

6. Nadeem S, Sadaf H (2017) Exploration of single wall carbon nanotubes for the peristaltic motion in a curved channel with variable viscosity. J Braz Soc Mech Sci Eng 39:117-125

7. Shahzadi I, Sadaf H, Nadeem S, Saleem A (2017) Bio-mathematical analysis for the peristaltic flow of single wall carbon nanotubes under the impact of variable viscosity and wall properties. Comput Methods Programs Biomed 139:137-147

8. Ijaz S, Shahzadi I, Nadeem S, Saleem A (2017) A clot model examination: with impulsion of nanoparticles under influence of variable viscosity and slip effects. Commun Theor Phys 68(5):667

9. Ellahi R, Rahman SU, Gulzar MM, Nadeem S, Vafai K (2014) A mathematical study of non-Newtonian micropolar fluid in arterial blood flow through composite stenosis. Appl Math Inf Sci 8:1567-1573

10. Hayat T, Nadeem S (2017) Aspects of developed heat and mass flux models on 3D flow of Eyring-Powell fluid. Results Phys 7:3910-3917

11. Hayat T, Nadeem S (2018) Flow of 3D Eyring-Powell fluid by utilizing Cattaneo-Christov heat flux model and chemical processes over an exponentially stretching surface. Results Phys 8:397-403

12. Ijaz S, Nadeem S (2017) A balloon model examination with impulsion of $\mathrm{Cu}$-nanoparticles as drug agent through stenosed tapered elastic artery. J Appl Fluid Mech 10(6):1773-1783

13. Ijaz S, Nadeem S (2017) A biomedical solicitation examination of nanoparticles as drug agents to minimize the hemodynamics of a stenotic channel. Eur Phys J Plus 132(11):448

14. Saleem S, Nadeem S, Sandeep N (2017) A mathematical analysis of time dependent flow on a rotating cone in a rheological fluid. Propuls Power Res 6(3):233-241

15. Mitsoulis E (1986) Fluid flow and heat transfer in wire coating: a review. Adv Polym Technol 6(4):467-487

16. Bagley EB, Storey SH (1963) Share rates and velocities of flow of polymers in wire-covering dies. Wire Wire Prod 38(8):1104

17. Khan NA, Sultan F, Khan NA (2015) Heat and mass transfer of thermophoretic MHD flow of Powell-Eyring fluid over a vertical stretching sheet in the presence of chemical reaction and Joule heating. Int J Chem Reactor Eng 13(1):37-49

18. Mahanthesh B, Gireesha BJ, Gorla RSR (2017) Unsteady threedimensional MHD flow of a nano Eyring-Powell fluid past a convectively heated stretching sheet in the presence of thermal radiation, viscous dissipation and Joule heating. J Assoc Arab Univ Basic Appl Sci 23:75-84

19. Khana N, Sultan F (2016) Homogeneous-heterogeneous reactions in an Eyring-Powell fluid over a stretching sheet in a porous medium. Spec Top Rev Porous Media 7(1):15-25

20. Hayata T, Aslam N, Rafiq M, Alsaadi FE (2017) Hall and Joule heating effects on peristaltic flow of Powell-Eyring liquid in an inclined symmetric channel. Results Phys 7:518-528

21. Sadaf H, Nadeem S (2017) Analysis of combined convective and viscous dissipation effects for peristaltic flow of rabinowitsch fluid model. J Bionic Eng 14(1):182-190

22. Ahmed A, Nadeem S (2017) Biomathematical study of timedependent flow of a Carreau nanofluid through inclined catheterized arteries with overlapping stenosis. J Cent South Univ 24(11):2725-2744

23. Nadeem S (2017) Biomedical theoretical investigation of blood mediated nanoparticles $\left(\mathrm{Ag}-\mathrm{Al}_{2} \mathrm{O}_{3}\right.$ /blood) impact on hemodynamics of overlapped stenotic artery. J Mol Liq 24:809-819 
24. Ahmed A, Nadeem S (2017) Effects of magnetohydrodynamics and hybrid nanoparticles on a micropolar fluid with 6-types of stenosis. Results Phys 7:4130-4139

25. Rehman FU, Nadeem S, Rehman HU, Haq RU (2018) Thermophysical analysis for three-dimensional MHD stagnation-point flow of nano-material influenced by an exponential stretching surface. Results Phys 8:316-323

26. Muhammad N, Nadeem S, Mustafa MT (2018) Impact of magnetic dipole on a thermally stratified ferrofluid past a stretchable surface. Proc Inst Mech Eng Part E J Process Mech Eng 1:1989-1996

27. Sadaf H, Akbar MU, Nadeem S (2018) Induced magnetic field analysis for the peristaltic transport of non-Newtonian nanofluid in an annulus. Math Comput Simul 148:16-36

28. Nadeem S, Ahmad S, Muhammad N, Mustafa MT (2017) Chemically reactive species in the flow of a Maxwell fluid. Results Phys 7:2607-2613

29. Rehman AU, Mehmood R, Nadeem S (2017) Entropy analysis of radioactive rotating nanofluid with thermal slip. Appl Therm Eng 1122:832-840

30. Muhammad N, Nadeem S (2017) Ferrite nanoparticles Ni$\mathrm{ZnFe}_{2} \mathrm{O}_{4}, \mathrm{Mn}-\mathrm{ZnFe}_{2} \mathrm{O}_{4}$ and $\mathrm{Fe}_{2} \mathrm{O}_{4}$ in the flow of ferromagnetic nanofluid. Eur Phys J Plus 132(9):312-321

31. Ijaza S, Nadeem S (2018) Consequences of blood mediated nano transportation as drug agent to attenuate the atherosclerotic lesions with permeability impacts. J Mol Liq 262:565-575

32. Rashid M, Shahzadi I, Nadeem S (2018) Corrugated walls analysis in micro channels through porous medium under electromagnetohydrodynamic (EMHD) effects. Results Phys 9:171-182
33. Akbar NS (2017) Double-diffusive natural convective peristaltic Prandtl flow in a porous channel saturated with a nanofluid. Heat Transf Res 48(4):283-290

34. Shahzadi I, Nadeem S (2017) Impinging of metallic nanoparticles along with the slip effects through a porous medium with MHD. J Braz Soc Mech Sci Eng 39(7):2535-2560

35. Rehman FU, Nadeem S (2018) Heat transfer analysis for threedimensional stagnation-point flow of water-based nanofluid over an exponentially stretching surface. J Heat Transf 140(5):7

36. Ahmed A, Nadeem S (2017) Effects of single and multi-walled carbon nano tubes on water and engine oil based rotating fluids with internal heating. Adv Powder Technol 28(9):1991-2002

37. Mehmood R, Nadeem S, Saleem S, Akbar NS (2017) Flow and heat transfer analysis of Jeffery nano fluid impinging obliquely over a stretched plate. J Taiwan Inst Chem Eng 74:49-58

38. Rehman FU, Nadeem S, Haq RU (2017) Heat transfer analysis for three-dimensional stagnation-point flow over an exponentially stretching surface. Chin J Phys 55(4):1552-1560

39. Hayat T, Nadeem S (2017) Heat transfer enhancement with Ag$\mathrm{CuO}$ /water hybrid nanofluid. Results Phys 7:2317-2324

40. Muhammad N, Nadeem S, Haq RU (2017) Heat transport phenomenon in the ferromagnetic fluid over a stretching sheet with thermal stratification. Results Phys 7:854-861 